Témoigner Témoigner. Entre histoire et mémoire

Getuigen Revue pluridisciplinaire de la Fondation Auschwitz

$117 \mid 2014$

Amis ? Ennemis ? Relations entre mémoires

\title{
Jacques Le Rider, Les Juifs viennois à la Belle Époque
}

\section{Colette Gutman}

\section{Q OpenEdition}

\section{Journals}

\section{Édition électronique}

URL : http://journals.openedition.org/temoigner/822

DOI : 10.4000/temoigner.822

ISSN : 2506-6390

\section{Éditeur :}

Éditions du Centre d'études et de documentation Mémoire d'Auschwitz, Éditions Kimé

\section{Édition imprimée}

Date de publication : 1 mars 2014

Pagination : 132-133

ISBN : 978-2-84174-663-7

ISSN : 2031-4183

\section{Référence électronique}

Colette Gutman, « Jacques Le Rider, Les Juifs viennois à la Belle Époque », Témoigner. Entre histoire et mémoire [En ligne], 117 | 2014, mis en ligne le 01 juin 2015, consulté le 23 octobre 2020. URL : http:// journals.openedition.org/temoigner/822 ; DOI : https://doi.org/10.4000/temoigner.822 
Bref une mise au point très éclairante relative à la Shoah, adaptée à un public spécifique.

Par le biais de l'aventure familiale ici contée, c'est donc bien au Sobibor mis en place dans le cadre de dont il est question. Heydrich que nous retrouvon cisement au centre du roman de Laurent Binet.

Fixé à Prague, ce haut dignitaire nazi, I'un des responsables les plus importants de la «solution finale », est présenté viales quate Himites des mots composan lexp ent signifiant par ailleurs: Le cervou d'Himmler s'appelle

Ce dernier sert aussi de pivot à une action dont le déroulement peut se résumer ainsi : Jozef Gabcik et Jan Kubis, deux résistants, un Tchèque et un Slovaque, partis de Londres et parachutés près de Prague pré pa 27 mai 1942 mais la mitraillette de l'un 'onraye le 27 mai 1942, mais la mitraillette de lun s'enraye et la grenade de lautre natteint pas l'Allemand. Blessé néanmoins, celui-cimeurt dune septicemie huit jours plus tard. Trahis et traqués eux-mêmes par la suite, le deux exécutants se suicident dans une église où ils se Interents Indépendamment del'intérét que comporte le rappeldeséven danslesinter qui sont animés par le souci constant déviter le romanesque facille grace à une dénarche singulière de fictionnalisationet à respect du passé: J J espère simplement que derière lépaisse couche réfléchissante d'idéalisation que je vais appliquer à cette histoire fabuleuse, le mirir sans tain de la rélice historique se laissera enco

Revendiqué ainsi d'emblée, cet équilibre fragile et scrupuleux se maintient remarquablement jusqu'au terme du livre ou il se trouve à nouveau convoqué «Mon histoire touche à sa fin et je me sens complètement vide, pas seulement vidé, mais vide. Je pourrais marrêter là, mais non, ici, ça ne marchera pas comme ça. Les gens qui ont participe a cette histoire ne sont pas des personnages ou en-tout-cas sils le sont devenus par ma faut l f faudrait naturellement pouvir citer tels [p. 431]. In faudrait naturellement pouvoir cite l'encore- nombre de métalepses apparaissant dans 'entre-deux...

C'est presque comme en écho aux derniers mots de l'auteur «[...] moi aussi, peut-être, je suis là » [p. 441]
que semblent résonner le titre de l'écrit de Dominique l'Aktion Reinhard, en hommage à Reinhard Heydrich,

Sigaud et les pages qui suivent décrivant une rencontr maginaire entre lauteure et Stangl, ancien comman-

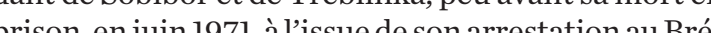
prison, enjuin 1971, al issue desonarrestationau Bré sllet de sa condamín à perpétuité. Ce tête-à-tête exceptionnel se déroule à lorigine dans louvrage de Gitta Sereny, Au fond des tenèbres (197), rapportant et destinés à rendre compte, ave l'appui de ténâ destiné à gnages de dépontés survivants, du régime en vigueur

Mais, élément capital en l'occurrence, c'est moins à cette situation que se réfere Dominique Sigaud quaux ches culpabilté » et « Je nail plus despoir » [p. 30-31] en forction desquelles elle ne peut pas ne pas voir surgir et son lecterinecte conner

D'où les partages observés, tournant autour des compromisetcompromissions dalors et daujourd'h à propos de la Shoah ainst que la menace denouvelles

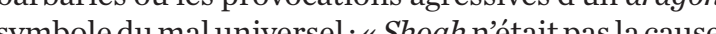
shooh n’était qu'un des visages, nis tas la cause, monde ext qu' des valets, peneurs et généroux assile têtes, servipar au départ des mères dévêtues et leurs ent avec calme au gez qu cestimera qui leur était demán quil leur était denandé, ne pouvaient donc s'adresser personnellonentán

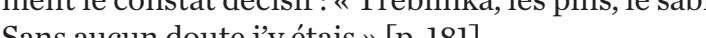
Sans aucun doute jy étais » [p. 181].

Ces nuances et ses différences, avec ses prolongements réels et potentiels qué nous venons de voir une même et terAlbert Mingelgrün révéler la vérité, ce qui entrân le sucided À la jeune fille alors d'assumer la nouvelle dans toutes ses dimensions, familiale et personnelle.. dans Lecture acompané qui Maniane Che de manière très pertinente à loccasion de quatre arrêts sur lecture correspondant à des moments charnières significatifs, suggere des lectures de textes apparentes et des films a voir tout en proposant des exploitation vaux d’écriture.

\section{DOLFI ET MARILYN}

François Saintonge

Paris, Grasset et Fasquelle, 2013, 287 p.

ous l'apparence inoffensive d'un récit d'anticipation, avec ses codes et ses extravagances, Dolf médiocrité hun propose une vision saísissante de Cédiocrite humaine, entre causticité et délicatesse. lo

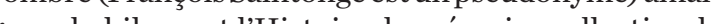
(a) humain.

Ce n'est pas, en effet, un humain lambda qui est symber d'un sè a rès sa mort, douze exemplaires ont éte dun sieccle après sa mort, douze exénplaires ont été puis frappés d'interdiction. L'un d'eux, premint d'une topés d'interdiction. L'un deux, premier lot principon pricu manque pas de piquant, et lauteur s'amuse beaucoup averti, qui découvre avec curiosité un « cas de figure imprévu de la condition humaine sur lequel sétait ju is condition hace chom prévisiblec lui quili sagit plutot d un cas de figure très previsiblaire décrivantle lot commun des clones depuis vocabulaire décrivantle lot commun des clones depuis terie, nourriture en croquettes, mise en service bomristion etc Ily a clairement de la part de l'auteur, une volon de cur à tront, lá clonage et objets d'un nouveau marché très lués par Cette approche, assez classique, a déjà donnélieù̀ fictions bien plus dérangeantes, comme le marnifice fictions bien plus dérangeantes, comme le magnifique roman de Kazuo Ishiguro, Never let me go. Saintonge de nous imposer en guise de clones, de véritables de nous imposer, en guise de clones, de véritables de Philippe Hamon. Si son Adolf Hitler et sa Marily de Philippe Hamon. Si son Adolf Hitler et sa Marilyn Mons pourt quasi-câceaturaut, is n'en sont pas ridiest porté sur eux grâce à lintelligence du regard qu rédemption du monstrueux qui est en chaque homme, 
la seconde permet d'explorer certaines facettes du sentiment amoureux. À travers lui, surgissent des images comme autant de jalons mémoriels conduisant peu à peu à un renversement ironique de l'Histoire. C'est en effet Hitler, ou plutôt son clone, qui est poursuivi pa es Juifs ou torturé par des sadiques. C'est Hitler qu porte un matricule tatoué sur sa peau. Cest Hitler que tion tris secret, entouré de barbé ét équipé d'une

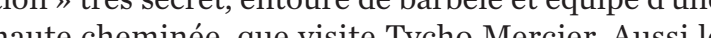
haute cheminée, que visite Tycho Mercier. Aussi le

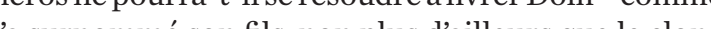
lán dégal de Marilyn quiéch

Du fait qu'il abrite ces deux clandestins, Tycho Mercier aurait pu être présenté comme un nouveau Juste, mais limage aurait été trop facile et l'on aurait la possibilité d'un retour de l'hor dèn travà rebours

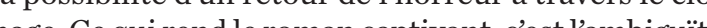
hage. Ce quirend le roman captivant, c'est lambigute du personnage principal. plein de bons sentiments et pon yn mais ne peut 'm lyn, nas ne dés àr.

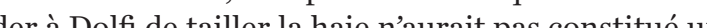
cer à Dolfi de tailler la haie n'aurait pas constitué un crime contrelhumanité», et seul le regard des voisin (c) forme desclavagisme légal. La petitesse du personnage ous rence com d'his est nócriture d'un livre qu'il ne teristoire englue quelcue quelque peu aigi par son divore, hais soncienx bouneur et ducapital soleil de son fils, ce pounrait être l'évolutiourrait étré evolution des pensées du personnage, ses doutes, ses sursauts de culpabilité, ses petits bonheurs. Il finit par syattar confidence, à la fois intime et spirituel, que Saintonge

Un homme ordinaire, donc, à la suite duquel le ecteur bascule dans le rocambolesque en abordan la seconde partie du roman. Au bout d'un mois de chation police. Après une ellipse temporelle de cinq ans, nous les retrouvons dans une petite principaute creee au nazi, nostalgique et mégalomane, lequel a conditionné

Dolfi pour en faire un Führer plus vrai que nature. On peut être déçu par cette évolution de l'intrigue qui, sans êt rimation des clones, « dès l'origine démunis, élevé vrai libre précieux bétail, par avance dépossédés d'un

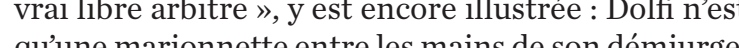
qu lecteur trouvera donc peu dintét à la desiption de $\mathrm{HJ}^{\mathrm{e}}$ Reich de pacotille, avec a la description de ce III Reich de pacotille, avec son apparat, ses costumes, ses drapeaux. Par contre, c'est encore une fois la personnalité de Tycho Mercier, toujours au

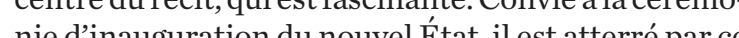
nie dinauguration du nouvel État, il est atterre par ce qu il y découvre, passant de la répugnance à la colère, drénétiquate à lefroi. Il finira pourtant par salue tendu à côté de SS en uniforme, et pau Fuhrer, bras tendu a côté de SS en uniforme, et par hurler "Sieg Heil! » avec eux. Le ronan de Saintonge nous inter-

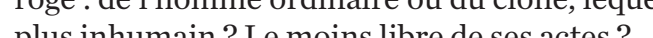

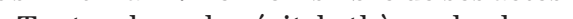

lout au long du récit, le the de du clonage permet humain. Tou les los Marlyn sonicite de chaque être mais cette idestité narilyn sont identiques, certes, dans le regard de Tycho Mercier, amas en singularite dans le regard de Tycho Mercier, amoureux de «sa » Marilyn ? Quel paradoxe, dailleurs, que ce signifian tité est ce qui fonde contre indivires, puisquelidenpuiss ce qui fonde notre individualté bien qu'elle puisse par aille ! se so entre les etres! Le clone incarne parfaitement cette antitise intrinseque et lauteur en joue à merveille,

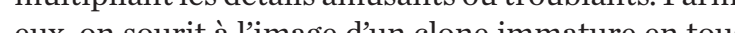
eux, on sourt à limage d'un clone immature en tous dém in tique à Herter parson démarque par une gourmandise incongrue. Saintonge n'est certes pas le premier a avoir imaginé le clonage dAivolf Hitler. En 1976, Ira Levin, dans Ces Garçon qui venaient du Brésil, proposait déja une intrigue dans laquelle Mengele lui-méne, après avoir fait naître 94 clones, contrôlait les événements majeurs de leur enfance, esperant ainsi voir eclore la personnalité du Führer... Quant a imaginer un Adolf Hitler totaledans La Part de l'autre enmanuel Schmidt s'y est risqué dans La Part de lautre en 2001. Dans ces deux fictions, évén événements externes dans la construction d'une personnalité. En ce qui concerne Dolfi et Marilyn, c'est individus: la Marilyn de Tycho devient unique aux yeux de son amant tandis que le Dolfi serviable et amical se métamorphose en graine de dictateur selon les visions d'un fou.

Bien documenté sur le plan historique, Dolfi et Marilyn est donc un roman qui se veut léger et distrayant, sans pour autant céder à la complaisance. Autant que la non-humanité des clones, c'est en effet l’’humanité des non-clones qui y est sans cesse questionnée, à travers une écriture en miroir fondée pour une part sur notre mémoire collective.

Karine Roy

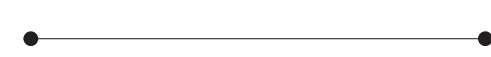

\section{KINDERZIMMER}

Valentine Goby

Paris, Actes Sud, 2013, 224 p.

avensbrück est un camp sans archives, mais

survivance de la parole des temoins a permis "écrire dans les silences de l'histoire », mais pour manifester « l'instant présent » d'une expérience qui manifester "linstant présent» dune expérience qu 1945, une Kinderzimmer (une poupo

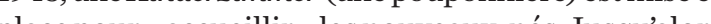
place pour « accueillir»lesnouveaux-nés. Jusqu’alor

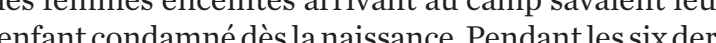
enfant condanódes la nains

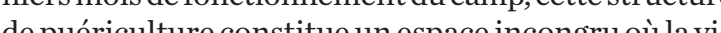

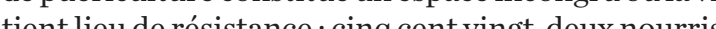
tientlieu désistan

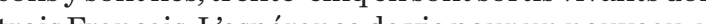
trois Français. L'espéra y etait de trois mois.

Après huit romans, Valentine Goby s'est emparé de cette particularité de l'histoire qui l'a habitée pendant trois ans. Elle fait tout d abord la connaissance dun rescapé. Un rapide décompte lamène à constate qu'il ne pouvait qu'y être né. H lui apprend lexistence bartde Lon (Sabiot lui presente Marie-José Chommédecin (Sabine dans lesonan), alons toute jeune médecin de vingt ans à la Kinderzimmer. D autres resCes tém et les récits de survivants constituent le cœur du roma de Valentine Goby : toute la Kinderzimmer est écrite au plus près de la parole des déportés. «L'imagination ferait moins bien. Mon but est de servir le témoin. [... Tout ce que je sais sur la Kinderzimmer, je l'ai mis

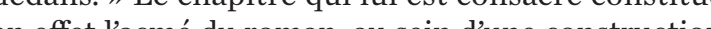
en éche

Valentine Goby tisse son personnage principal, Suzanne Langlois/Mila (nom quelle prend dans camp), des difres/sts fils qui lui ont permis de se figud'une femme vulnérable, simple, aux antipodes de d'une fenmme vulérable, simple, aux antipodes de cuerre, nont laisś aucune place au doute: guerre, n'ont laissé aucune place au doute : celles qu axquelles on a du mà à s'identifier L sur le témoign nul suzanne Langlois, ran sóe ure le temange que Suzanne Langlois, rescapee de Ravensbrick, présente à unjeune auditoire. Le lecteu, plonge alors danslexpérience de Suzanne/Mila narrèe à la troisième personne du singulier : cette distanciaton place néanmoins lelecteur au cœur de lexpérience Mila décourre la réalité du camp alors quelle pro/ peu a peu conse la realite du canp alors quelle prend peu à peu conscience du fait quelle est enceinte. Face du dedan à l'apprentissage de cette nuver du dedans » : à lapprentissage de cette nouvelle langue - "le camp est une langue » qui renvoie la déportée a la déc lingans ne disposant pas de la parole-sajoute

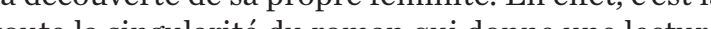
féminin dur peminine du camp. Limage du corps qui demeure un percò

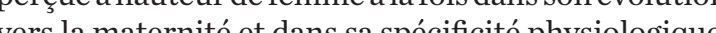
vais le corps n'incarne pas uniquement lasiologique. ingulière vécue par Mila : il manifeste le lieu d'u réconfort pesse par Mila : il manifeste le lieu duu réconfort possible dans lamitie que Mila et Teres des transerts affectifs que Valentine Goby parvient a rendre do foction pointilliste, par petites touches à rendre, de façon pointilliste, par petites touches de de Michel Foucault «Del'amitié comme muer le texte

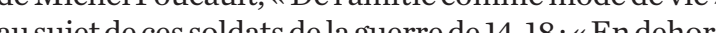
de quelques propos sur la camaraderie, la fraternité d'âm, de propos sur la camaraderie, la fraternité d'âme, de quelques témoignages très parcellaires, que coeur quil y a pu y avoir dans ces mes dempêtes de cour qu il y a pu y avoir dans ces moments-là ? Et on peut se demander ce qui a fait que, dans ces guerres 
ont malgré tout tenu. Par un tissu affectif sans doute.» On peuts'interroger sur la nécessité de donner une

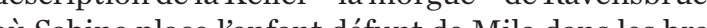
on morts dune mere. Valentine Goby explique alors que cest un charnier epouvantable qui devient un abri, faut défigurer ce rell pour venir vers cette inage-là,

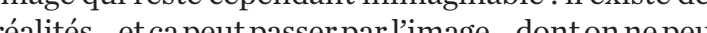

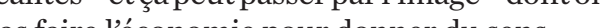

Sharlotte Delboet d' chasse l'expérience des canps, elle parvient à sinspar le choix du point de vue natatif et le détor de par le choix du point de vue n. fiction, et très proche par le cisellement dun style qui reste à fleur de peau. Le ronan, selon Valentine Goby, pésent Mais plus qu'une figuration de l'inistant présent. m Mais plus qu'une figuration de l'ininagnable, il en Mass plus quage

Isabelle Galichon

\section{LES JUIFS VIENNOIS
A LA BELLE EPOQUE}

Jacques Le Rider

Paris, Albin Michel, 2013, 354 p

ienne, capitale de la modernité au XIXe siècle, puis lieu de triste mémoire, prélude à la Shoa Ses mutations dénographiques lont dabord transformés en "Jérusalem delexil», avec lanflux de l'mmigrés juifs de l'est - Ostjuden - qui, ayant acquis légalite des

Cela se passe dans les années 1880. Les Juifs viennois de vieille souche, assimilés et intégrès, découvren alors une autre identite juive - un choc des culture qui leur semble étrangère, voire exotique. Cest à ce dans tous les milieux de la société viennoise en véri- table code culturel. En 1897, Vienne est la seule grande ville européenne gouvernée par le parti antisémite vec léection de Kart

Un rabbin, Samuel Bloche, s'engage dans les lutte ociales et le combat contre l'antisémitisme. Il invente la formule « Autrichiens de nationalité juive ».

Mais Nathan Birnbaum et Theodor Herzl opposent odèle judéo-viennois en crise celui de la « désas(1) fication d'un Etat-nation juif. Ce n'est pas vraiment la position de Freud. « Jai assurément les meilleurs sentinents de sympathie pour des efforts librement etje me réjouis de la prospérité des ét de Jérusalem

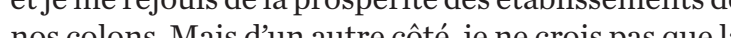
nos colons. Mais dun autre coté, je ne crois pas que la Palestine puisse jamais devenir un État juif ni que le un jou ĉtre prêts à confier leurs lieux sinte puissent un jour êtrep

Enfin s'ouvre une autre voie avec Victor Adler et Otto Bauer, celle de l'engagement socialiste.

Antisémitisme généralisé quasi officiel, sionisme socialisme, exils : tout est dit, tout est prêt pour les

Sigmund Freud et son double littéraire, Arthur Schnitzler (qui se définit comme «Juif, Autrichien, Allemand »), ouvrent le sieccle aux chefs de file de la jeune Vienne, Hugo von Hofmannsthal, Richard BeerOtto Weininger, St chönberg.

Arthur Schnitzler, dans son monumental Journal personnel, suit au jour le jour la prise de conscience de son identité juive et exprime sa « fureur contre la bassesse humaine » qu'il aperçoit dans les diverse formes de judéophobie. Son identité juive est une affaire personnelle "[...] le fait que je sois venu au ter Si des millions de crétins trouvent que je n’ai pas ter. Si des Aillic je de mieux que ceux-là que je suis ic ma place ici, je sais mieux que ceux-là que je suis ici de l'Autriche et de Vienne est aujourd'hui ressentie de lAutić et de Vienne est aujourd huif ressentie et exprimée avec plus de force par les Juifs que par les antisémites. Et sice million trouve que je ne suis pas chez noi ici,je rétorque que pour moi, hien d autre ne compte que mon sentiment personnel. » Sa pièce, $L a$ Ronde provoquera un scandale à la Première et sera juif $\gg$.
Stefan Zweig, «bon Européen», citoyen du monde, issu de la bonne bourgeoisie juive, éprouve de la compassion pour les « petits » Juifs de Galicie qu'il a vu arriver et dont il a décrit le tragique destin dans $L$ Bouquiniste Mendel. L'écrivain à succès ne se sent pas personnellement concerné, il pratique l'humanisme cosmopolite et tolérant d'Érasme et de Montaigne, se considère comme un citoyen du monde. Sa vraie patrie est Europe, modalité de l'universel humaniste, aboutissement d'un destin historique partagé, civilisation idée diversite, respect des différences. « Je tiens les

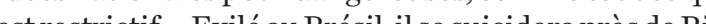
estrestrictio

Des écrivains aussi différents que Stefan Zweis Joseph Roth et Robert Musil nauront eu comme point connu quis deleur te de la écen de la «montée en puissance dune nouvelle barbarie. Gustav Malher est né dans une famille juive assinilée à la culture aleséd estele Juif Malher, directenr de lopera, sa nusique est allemande - avecun ac et de la mélancolie...

Le poète Elias Canetti a érigé un monument à la mémoire d'Abraham Sonne, rencontre à Vienne, dan ses Jeux du regard. Abraham Sonne est « un homme à Vience at de la perterin. Temon de

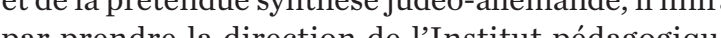
par prendre la direction de l'nstitut pédagogique Juifs vien de sè leur dép dert pour la Palestine. C'estes

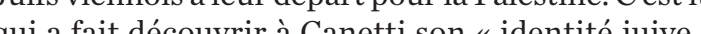
qui a fait découvrir a Canetti son «identité juive parce que den dre vie noit être nién

la Vienne annexée par Hitler,la Vienne à léquil

Colette Gutman

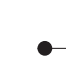

-

\section{LES CAMPS NAZIS. RÉFLEXIONS SUR LA RECEPTION LITTÉRAIRE FRANÇAISE}

Yannick Malgouzou

Paris, Classiques Garnier, coll. «L Littérature, histoire politique », 2012, $616 \mathrm{p}$.

une véritable prise en considération ment que l'ouvrage de Yannick Malgouzou, issu d'une thèse soutenue en 2007 , nous ans toute son épaisseur, c'est-à-dire, paradoxalechent, de l'én épaisseur, c'est-à- dire, paradoxaleque la liéránen ambitionné d'en restre compte. À travers mentlioneé den rendre compte. Átravers notamde l'après-guerre, Les Camps nazis. Réflexions sur la dé laprès-guerre, Les Camps nazis. Reflexions sur la cecpton litter elle fut fractionnée) des débats autour du statut des émo fut fractionnes des débats autour du statut des pouruser dela Dour

La dimention

La dimension transversale de lapproche de $\mathrm{Ma}$ détour lautor lế notamment à opérer un important tetion testimoniale dès avant la fin de la guerre, pouss à voironiale dès avant la fin de la guerre, et tion de l'écriture du témoigne a : le témoignage tel

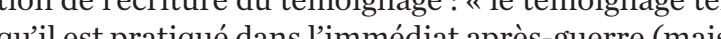
quil est pratiqué dans 'immédiat après-guerre (mais

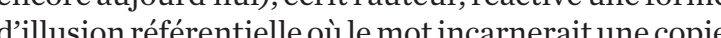
trit térale» [p 161]

Mais l'intention des témoins consistant à « donner a voir » [lexpression est de Charlotte Delbo : cf. la (p. VII) et l'inadrion de in Charlotte Delbo, Auschwitz and After, traduction Rosett $C$. Dello 1995] l'. Lánont, New Haven, Yale University Press, l995] lexpérience concentrationaire au travers de contre la photographie, c'est-à-dire avance lauteur, une forme de sensationnalisme; contre, ausi, une 
évidence en apparence immédiate qui caractérise par définition l'ordre du visible. La lite

Se confronter à la photographie, à la pratique orale $\mathrm{d}$ témoignage, réfuter ou dialectiser les discours sociaux tenu témoignage, réfuter ou dialectiser les discours sociaux tenu sur les camps ne sont jamais que lexpression dune pratique

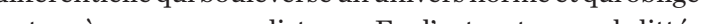
lecteur à mesurer une distance. En dautrestermes, la litter qui se construit contre l'inimaginable [p. 145].

Le projet littéraire vise à la fois à donner à voir et à direl'incom ment decrit. Lessai de Malgouzou entreprend l'étud de cette triple rupture (de la contemporaneité, de lordre du voir. lo a place àla votion in le sen du

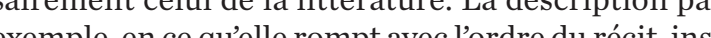
exemple, en ce quélle rompt avec lordre du récit, inscifo qui fait la distanceentrele lecteuret Kanteur plus sidérante à mesure que celui-ci s'efface derrière de purs présentatifs et que la scène décrite semble comm

La prése du d́cit, ò le tém

La présence du décrit, ou le tèmoignage paraît se décharger du sens qu' inpose la mise en récit, suscite une forme décniture de la mise à distance dont Magouzoú releve qu'elle n'est pas étrangère, dans sa nouveante, à la conceptualisation par Roland Barthes du d'un resó [en l'ost par le truchement des textes dûn rescapé [en loccurrence Jean Cayrol], risque définit ulgouzou, que Rolán barthes élabore et céchin hypothèse est notamment renforcee par le regard rétrospectif que porte Claude Simon sur le mouvement à la fin des années 1980 et dont ussi profondement les enjeux.

Ilest pourcette raison dommage quau lieu de tire cette ligne qui donne veritablement à lévénement France (et par là définit peut-être plus largement voince (et par là défnit peut-être plus largement les voies par lesquélles la litterature fraye avec le reeel) Réflexions sur l'usage de la référence qui concluent Réflexions sur lusage de la rérérence » qui concluent velle introduction inscrivant son travail dans le champ des Cultural Studies. L'analyse d'œuvres littéraires le cédait déjà, quelques pages plus tôt, à celle d'objets littéraires comme Acide sulfurique (2005) d'Amélie Nothomb [p. 377 sqq.] sur lequel l'auteur suspend son jugement pour en faire l'indice de l'intégration d'Auschwitz dans la culture de masse, tout en soupçonnant en note qu'il s'agisse d'une œuvre de circonstance. La quatrieme partie s'ouvre donc sur un long déveloptitué pou lasitions de Claude Lanzmon et de titue par les positions de Claude Lanzmann et de se xégètes sur Shoah ittéraire du sujet.

ll est d'ailleurs significatif qu’à l'exception de quelques retours du littéraire, comme la mention des Bienveillantes (2006) de Jonathan Littell, ce soit par tion " de chèma que les thèmes de la "trivialisa-

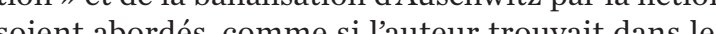
cilms anordes, cons flins un domaine plus nettems livres. Lintention prescriptive qui semble animer les cincer tistion culturelle d'un événem cet [s] déqualifcation [Régis Debray, Cours de métiont eque déqualification " [Régis Debray, Cour de médiologie générale, Paris, Gallinard, coll. " Folio essais », 1991, p. 300], [p. 476]. Levenenement passan a loeure et lomble pouvori etre à chaque fois qu'une dégradation dont le seul garde-fou serait qu' « il reste néanmoins possible de constituer la Shoah en objet détude pour circonscrire ses modes de fonction Plutats » [. 477].

Perspective, Malgouzou décrit en fait ici, à peu de choses près, l'état actuel du rapport de la culture à Auschwitz. En cédant sur le sujet a d hypothétiques attentes que nourrirait un public lieu de susciter de véritables critiques, provoquent lieu de susciter de veritables critiques, provoquen le plus souvent tire pure et simple des universitaire devant le manque flagrant de serrieux dans la manière dont cescentenent. Or on est tout autant las des colères universitaires qu leurs anathèmes une morgue de classe pour lancer leurs anathèmes que de la grossièreté des procédé dont certains écrivains et artistes usent pour évoque Auschwitz. Le public, pour sa part, quand il n'adopte pas a son tour la gravité du specialiste ou qu'il n'ose y a pris, reste un peu confus de sa crédulité et s'of- fusque qu'elle ne leur soit pas pardonnée, menaçant à mi-mot de se désintéresser à la fin du sujet. On sait rinextricabledecettesituationà aquelle lessurvivan 'insticter de plus en plus précaire

rapport de la culture à Auschwitz Pende une autre perspective et requiert surtout (tart ne se laisse pas dome tiquer, et que c'est précisement parce que l'art défie ce qu'on attend de lui qu'il est en mesure, si peu que pour, reprendre l'expresion de l'auteur, sans a pour reprendre lexpressin de l'auteur, sans quă

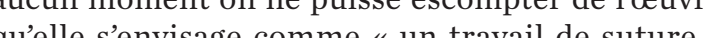
[p. 479] de cette p. 479] de cette mêne béance. L'aphorisme de René Char que cite Malgouzou dit assez combien l'art sait être in

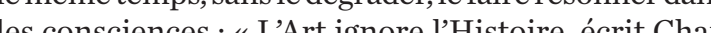
les consciences: « LArt ignore lHistoire, ecrit Char, recherche de la base et du [1955] Paris, Galli-

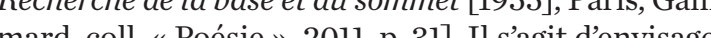

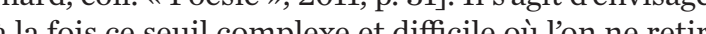
a pas plus a l'effrayant de l'art qu'on ne retranche de so

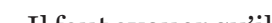

Taut avouer qu'il est malaisé de se faire de ce seuil une idée, et reconnaître que si parfois il semble vouloir y parer, Malgouzou ne l'ignore pas puisque, ave dém, il a d abor. 102]. Dans le contexte act démarcation $\gg[\mathrm{p} .102]$. Dans le contexte acámique de désacralisation des notions dinnaginable et dirleuteur dévénet l'évìn

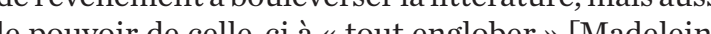
le pouvoir de celle-ci à «tout englober» [Madeleine Chapsal, "Rien que des fenmes. Entretien avec Char-

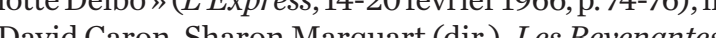
Charlotte Delbo La Voix d'une commes hé à jutes. Charlotte Delbo. La Voix dune communaute à jamais coll Cribles. Essais de littérature 2011, p 23] coll. « Cribles. Essais détitter ature », 2011, p. 23].et, mutatis mutandis, à reiterer par les nots le dérangecette fois vis-à-vis de l'histoire de la litté ture même, reste difficile dérane, re diffillement appréhensible parce quau

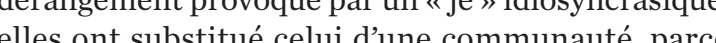
qu'un « nous » confondant les vivants et les mort s'inscrit désormais au cour du récit, et que ce simple chement d'énonciateur dit assez pourquoi tout écrit sur Auschwitz porte au-dela de littérature.

Paul Bernard-Nouraud

\section{LALITTÉRATURE \\ DES RAVINS}

pelboin, Assia Kovriguina,

réface de Catherine Coquio

Paris, Robert Laffont, 2013, 288 p.

our la Russie stalinienne, Kolyma, c'est la 《erme que Dachau ou Auschwitz pou IAllemagne hitlérienne. Ni Yune ni l'autre ne pourront jamais se débarrasser de ces noms. Tolyma le mas pronsel qui caracté coly contemporaine, de la même façon que dans les fours Introl Intiod

Dans le parallèle énoncé par l'ancien zek Siniavski qui préfere nommer Russie staliniennel'Union soviétique, livre nul de place pour « les ravins ». Au terme de ce universel $»$, ont une part très particulière. 'est nile camp d'extermintion etde déportation nist nile camp dexter in physique quand le, ouvert qui relève de la gèographié pynépácicis de La Litter ature des ravins ne manquent pas de de La Littérature des ravins ne manquent pas de souligner une difference majeure : la population voit, car étudest tém to études, témoignages sur les camps nazis et le Goulag les récits sur les camps de la Chine maoïste et postles rècits sur les camps de la Chine maoìste et postderiste des ravins. Pourtant, tout autant que les camps d'exterdes Juifs deur rave 
germano-soviétique rompu, les troupes hitlérienne envahissent Pologne et Union soviétique, massacren plus est celui de Babi Yar près de Kiev en Ukraine.

Annie Epelboin et Assia Kovriguina prennent méthodiquement le parti de faire tomber ce que cache la rhétorique hérö̈que d'un État (Union des républiques socialistes soviétiques) et d'un pouvoi détestés, assiégés par les troupes d'Hitler. La thèse du livre est que la stratégie idéologique stalinienne a pour but de donner forme à un effacement de la spécificité des massacres, en particulier de gommer leu caractère génocidaire et de fondre la Shoah au sein anonyme des millions de victimes de la guerre. Pour cela il faut construire et imposer l'idée que les tueries sur place, au vu et au su de la population - encore une fois différence capitale d'avec le camp - ne visent pas les Juifs en tant que Juifs, mais des citoyens/citoyenne soviétiques. Selon le langage officiel, des « civils soviéovertiques. Selon le langage officiel, des civils soviétesues » composent linmense majorité des victimes lánomination de la guerre ( gues. Cest pour cht pst un eu de preniere importance. Si la guerre est patriotique, toutes les victimes payentidentiquement eient juives ou non Est du soient juives ou non. Est du mêne coup aboli le trait exté systénatique ex territoires polon et soviétique Pour tant, au sur même la guerre, lorsque même de la guerre, lorsque commence le reflux des cest-à-dire pas soviétique - propre aux ravins et cest-a-dire pas soviétique - propre aux ravins et qu a mémo la mémoire et du passé. Le démenti, en quelque façon anticipé, se forge dabord en appui sur les tèmoignages de l'Armée rouge, les premire quis sont, avec les soldats delArmée rouge, les premiers a découvirir les « ravins

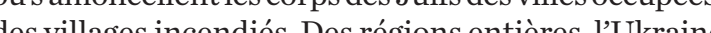
des villages incendiés. Des régions entières, I'Ukrain SS, de ainsi que le note Grossmann. Ces chroniqueurs de la guerre rapportent ce que des survivants et des lans Le Lirenir [Hya dans LeLivrenoir [lya Ehrenbourg, Vassili Grossman (textes etten Solin/Actes Sud, 1995, 1130 p.]. Le livre est probableLittell. Il rassemble, réunit les récits insoutenables des «actions », c'est-à-dire des atrocités, commises par les diverses unités de l'armée allemande. Reste le statut du Livre noir, autorisé, interdit pour ne paraître tel qưon peut le lire aujourd'hui quau moment de la Pereströ̈ka initiée par Gorbatchov.

Pourquoi faut-il, nier, passer sous silence, faire dispartre «la destruction des Juifs » d'URSS comme per er le rôle des populatione Pourquoifaut-il minimison de prêter main-forte aux mamandes, forcees ou non, de préter main-forte aux massacres? La réponse l'ouvre d'un antisémitisme d'État particule ello lo émitable sous Staline, lequel épouse sans peine lanti-

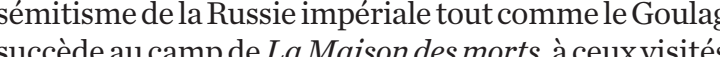
técrits

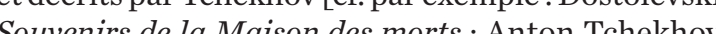
L'Île de Sa da Maison des morts; Anton Tchekhov, the de Sakhaline].Pour ce qui est del'Ukraine, la ven[Robert Conquest La Lrande Terreur précédé de SanTlantes Conquest, La Grande Terreur précédé de SanLeuquins », trad. : Claude Sauses du southere a lenvahisseur nazi.

La seconde partie de La Littérature des ravins révèle a un public qui ne lit pas le russe une littérature peu celle des corte en français qui n'est plus seulement celle des correspondants de guerre, quils soient prosateurs ou poètes comme Grossmann, Chklovski, d'écrivourg. Il s'agit de proses, de poèmes, œuvres decrivains tourmentés par ces massacres, dont ils écrits à des ép temps après les massacres, n'ont guère, soins longtemps après les massacres, n'ont guère, semble-t-il, circulé sous le manteau. De fait mal connues, même ile poène Babi Yar d Evtouchenko qui inspira sa 13 symphonie à Dimitric Chostakovitch a franchi les fronteres de URSS, ces cuvres n'ont pas euen Occident le internements pychiatriques contrain Goulag, de interints. Leur reconfaiso façon dont ils prèsentent les tueries, les supplices, les mectilite vec la ligne idéologique officielle qui a cours à telle ou

Qu'Annie Epelboin et Assia Kovriguina ajoutent à leur enquête quelques traductions, et sur plus d'un rêt de le précieuses infornations, augmente linté-

prend qu'un vaste champ de recherche est ouvert et bien ouvert Raison de plus pour absolumentconnaître ce livre. I

Michel Enaudeau

\section{$\bullet$}

GHETTOSTADT

LODZ ET LA FORMATION D'UNE VILLE NAZIE

Gordon J. Horwitz

Paris, Calmann-Lévy/Mémorial de la Shoah, 2012, 375 p.

n septembre 1939, Hitler envahit la Pologne. Lodz - se trouve alors enserrée dans la partie occiden-

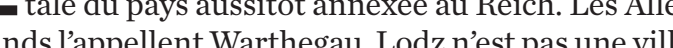

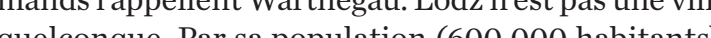
quelconque. Par sa population (600 000 habitants) elle est la seconde ville du pays. La vie intellectuel et artistique jouit d un reel renom. C'est aussi une ville ouvière. Les 200 oó Juifs qui y vivent forment la seconde communauté juive de Pologne. Beaucoup à la question: qu'est-ce qu' A la question : qu'est-ce qu'une ville nazie ? L réponse est sille: une ville vidée de sa population juive, « une ville sans Juifs ». La ville ethniquement tectes et urbanistes qui en recomposentloux archicectes en et les espaces. Cest à prenière vue la mise à plat des conditions etcirconstan les la nise en we de programme quiguide lenquête minutieuse et scrupuleuse de lhistorien a licin le devenir de la seconde ville de Pologne.

Faire de Lodz une ville nazie, c'est d'abord symboliquement germaniser son nom. Lodz devient Litzmannstadt, nom formé a partir du patronyme d'u generral, Karl Litzmann. Rues et places changent de doute : Anolf doute que cela ne saurait suffire. Le fanatisme de lhytion dur uneplaceinpression tion du regroupenent de la population juive et de son enferine plus finir les autorités conquérantes, c'est le fait des
Juifs. Débarrasser Lodz de la saleté, c'est débarrasser la ville des miasmes, des maladies, des risques d'épidémies (typhus, tuberculose), tous maux attribués à la puanter de la populationjuive qui n’a aucun souci des nolites de lingies hllow

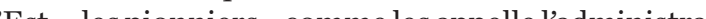
Est, lespion À com ton allemande. Aeux sont destinés, après rénovation, l'éviction des Juifs. En janvier 1940, soit un peu plus de trois mo Jì̀s l'invanion fficielle thise dé toú a L La/Litzan le tourné à Lodz/Litzmannstadt à la fin de lèté 1941 soit un an et demi après la création du ghetto, par des techiciens desstudios Universum Filn atte nazi. Le livre conmence dailleurs par la description Dans in Dansles dernières pages de Ghettostadt, Ihistorien fait réqur sur ce film inachevé qui s'attarde, entre autres . [p. 307].

L'historien accorde en fait peu de place à la reconfid' noc chapiste, "Le ghetto et la ville de lavenir », elle ville mutante, le sport, en particulier l'athétisms pos de loisirs de piscine brefd'un confort devie dui trance loc la ville qu'était Lodz avant son annexion Reich. Lessentiel de l'enquête soncerne subsion dans le

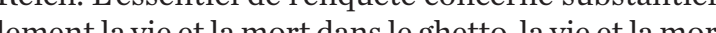
du ghetto, de son établiss lent (avil 1940) jur du ghetto, de son établissement (avril 1940) jusqu'

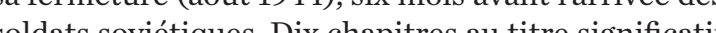
soĺats soviétiques. Dix chapitres au titre significatif ment organisées, affectées par le déroulement duegue Cette vie et cette mort assurent la e formation» dette vie et cette mort assurent la « formaHorwitz a pour lui la sûréé empirique et chail de gorwe co pour lui la sûreté empirique et chronololes dossiers d'une vaste documentation. Elle fournit les chiffres de la nourriture, rend compte de l'évintion démographique du ghetto indique l'état l'evolution démographique du ghetto, indique létat d'esprit de la police et du service d'ordre juifs, denombre les répertorie la production de bi ation pièce par pièce, 
régis par une organisation très surveillée du travail, consigne les rations alimentaires attribuées au fil de la che que re quelques concerts). Le point de vue nest jamais guidé

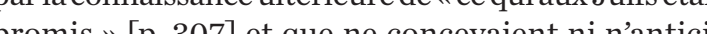
promis » [p. 307] et que ne concevaient ni nanticipaient les autorités juives inpliques par ladministhe lo lo jo place le ghetto. Hexige et obtient la collaboration des Conces juives dejà existantes, à connen conseiljuif, dontla pexsiden los

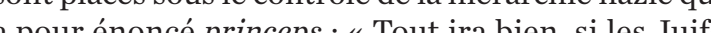
a pour énoncé princeps : «Tout ira bien, si les Juif copent. nas tont ne va pas bien et n'ira janais bien pour les Juifs de Lodz. Ça commence avant leur transfert complet dansleghetto, lorsque les Allemand . la ville. Dans la nut du 6 au 7 mars 1940 , environ deux cents sont abattus sur place, cent cinquante autres

Face à ces tueres, le ghetto relève pour certains de abri, du refuge, tel un lieu où en dépit de tout (malnutrition, froid, spoliation, etc.) la vie est maintenue. Le président du Consell juif impose avec une autorité pour foyer le travail. C'est épreuve une ligne qui a pour foyer le travail. Cest par le travail que les Juif

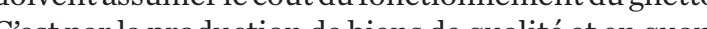
tité (objets de toute sorte, textiles pour l'ha en quantité (objets de toute sorte, textiles pour lhabillement, o que les Juifs resterontenvie au seindughetto. Chain Rumkowski n'aura de cesse que dexhorter à l'effor de travail pour satisfaire la demande sans cesse granthantede ladministration injontion injonctions - «travailler, travailler toujours plus », "travailler pour exister »- n'empêchent pas les prelo g du service d'ordre juif, les Allomo du service dordre juif, les Allemands organisent une e ghetto, on ignore cui le ghetto, on ignore ce qui se passe dans ce camp ouvert au gaz d'éc 1941. La mort y est donnée par asphyxie au gaz dechappenent de camions aménagés à cette ceux ignoré tout aut
Il n’est pas nécessaire de rapporter davantage l'information mise en ordre par Gordon J. Horwitz pour saluer l'apport de ce livre préfacé par Georges Bensoussan. Il complète considérablement les indications fondamentales déjà données dans les ouvrages d'Hilfondamentales déja donnees dans les ouvrages d'Hiliche juge pas, haccable pas le dur Ru Destruction des Juifs d’Europe tome 1 Paris Gellim, $L a$ Destruction des Juifs Europe, tome 1, Paris, Gallimard, "coll. Folio Histoire», 1991,p. 191]. Toutefois, lébranleles pages qui relatent l'impuissance de Rutent dan fes pages qui relatent linpuissa see de Runkowski face aux situations les plus difficles : soustraire les enfants aux Allemands. Faute de parvenir à empêcherla deportation, Runkowshiaccè aux demandes nazies : plus de travail, accepte de livrer des Juifs qui sexplique toujou a la nort loin du ghetto. Runk ju explique toujours que seul le sauvetage de vies juive arete ses décision. Cest, à lire Horwitz, lobsession unique et constante du president du Conseil juif. C'est pourquoi il sopposait par principe à un soulevement, quívolte àvait que provoquer une féroce réplique. Une réau à ses yeux aurait été dautant plus suicidaire quau contraire du ghetto de Varsovie, celui de Lodz ne détenait quasínent pas darmes. Sans mettre en pas être déconcerté par son aveuglem neut pas ne pas être déconcété par son aveuglenent politique et Juif de la Juifs de la surface de la Terre. Rumowski sera de ceux auxquels Hinmler, qui a décide la liquidation du ghetto de Lodz a interditle droit de "partager la planète». I Len a 1944.

La mort du ghetto signe la mort de la communauté juive de Lodz. Seul le principal responsable allemand de la gestion directe du ghetto, Biebow, est condamné à mort et exécuté en 1947. Bien plus nombreux sont ceux qui ont tapé sur la machine à écrire ordres et insrien n'est mo jue rien n'est pas un juge. Pas en charge, comme la écrit Chateaubriand, de la vengeance des peuples. Horwitz laisse son lecteur, intelligence et cœur, se débrouille avec ce qui demeure la Catastrophe ici à Lodz/Litz-
mannstadt.

Michel Enaudeau
GHETTOSTADT : LODZ ET LA FORMATION D'UNE VILLE NAZIE

e livre de Gordon J. Horwitz est une histoire chronologique de la ville polonaise de Lodz et de ses habitants pendant la Seconde Guerre mondiale.

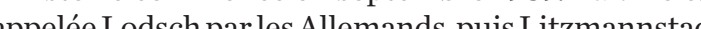
à pelée Lodsch par les Allemands, puis Litzmannstad a partir du 11 avil 1940, jusquá larnivée de lirmée rouge le 19 janvier 1945. En 1939, la ville comptait ( quartier de la ville tranforś été quartier de la ville transformé ghetto. Le livre prée la vie allemande d'une partet d'autre part la lente de la vie allemande d'une part et d'autrepror destruction, en quatre ans, de la vie juive.

Litzmannstadt devint vite une veritable cité expérimentale distes. Elle reçut la visite de Goebbels et dHime " ethniques " (Volksdeutsche) ont affué en raison d'un accueil en tout point parfait : logements, jardins. activités culturelles et sportives, musées, hôpitaux. vectucation et la for 27.6\% a a Mais abien-être des Allemands etleur saite $16,1 \%$. to et morale impliquaient une ville purifiee par la disp -

Cependant, les qualités professionnelles exceptionnelles des Juifs de Litzmannstadt, en particulie man à créer un ghetto rosiontant mands à creer un ghetto rassemblant plus de 164000 parré) desting carré) destine à exploitation du travailjuif. On a, bie entendu, retrouvé les tensions habituelles entre les fiter de quivoulaient faire tavailler los Juirset pro-

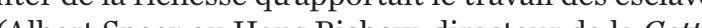
(Albert Speer ou Hans Biebow, directeur de la Gettode Himm, lad quinistration dire du ghe to hommes de Himmler qui voulaient faire du ghetto un camp de concentration entre lesmains des SS (par exemple Otto Quivisita Litzmanstadten décembre 1943). Mis tous quivisita Litzmannstadt endécenbre 1943). Mais tous voulaient plus ou moins rapice Iorsan

L'organisation juive du ghetto s'est trouvée entrele mains d'hommes qui espéraient, sans doute de bonn foi, sauver au moins une partie des Juifs par un travail intensif et si soigné qu'il serait indispensable aux Allemands. La collaboration du président du Conseil jui Mordechai Chaim Rumkowski, un personnage haute-

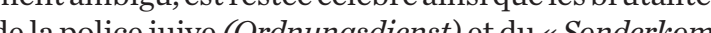
de la police juive (Ordnungsdienst) et du «Sonderkomde valeur caphés : méc les eftor de certaines des de vás juives pour mintenir un vie comes des autorités juives pour maintenir une vie communaue les

Lavie dus trofut marquée parlex La vie du ghettofutmarquée par lextrême violence nazie qui a alimentélangoisse pendant quatre années écu éé soumis les Juifs. Gordon Horwitz décrit la flambée de décidé de répétition avant de tuer les gens.

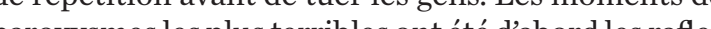
paroxysmes les plusterribles ont éte dabord les rafles des enfants (septembre 1942) et les déportations vers Kuls portations vers Auschwitz

Le récit de Gordon Horwitz est rendu particulièrement impressionnant par sa composition qui fait penser au langage cinematographique avec un montage en parallele des plaisirs allemands et des souffrances Juives, avec des plans densenble et des plans rapprotoire des estats est singuliem nent poignante : leur toire des enfants est singulièrement poignante : leur joie innocente de voir les premiers avions (allemands) en septembre 1939 ou leur plaisir inconscient de partir vers vers les camions à gaz de Kulmhof,le soin qu' ils apportèrent a confectionner les maisons de poupee pour les

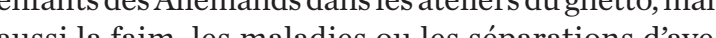
ussi la faim, les maladies ou les séparations d'avec Le livie ne signale auctse maniestaton de

Le livre ne signale aucune manifestation de compassion de la part des Allemands. La seule rèaction de la Marienkirche, l'église de Marie de Litzmanne que la ne soit pas incluse dans le ghetto (p. 62). On appren tions de ceux de ses clients qui n'auraient pes eortafini de payer leur machine à coudre achetée à crédit. f'administration machine a coudre achetée a crédit. Léglaninistration du ghetto a rassuré l'entreprise en
rectures sans problème avec l'argent volé aux Juifs [p. 174]. 
Pour un livre qui est excellent, il faudrait viser à 'excellence de la publication. La traduction comporte à plusieurs reprises l'emploi irritant à la manière des journalistes d'aujourd'hui de l'adjectif « palpable " pour dire manifeste, évident, sensible. Les " ponts reliant les différentes parties du ghetto sont plutôt des passerelles et les « projecteurs » qui servent à fildes passerelles et les «projecteurs » qui servent à fil-

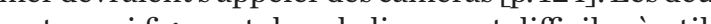
cartes qui figutier tirées d'un al live et le lecteur page 57. Elles sont tirees dun autre livre et le lecteur est quelquefois e peine pour retrouver le nom des rues dont il est quesique de cette importance, seuls les rento rque de cette inportance, seuls les renseignements cources et de la biblios donie et l'absen d'uns des

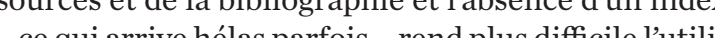
- ce qui arrive hélas part

Lelivrenefait pas appel aux derniers témoignages, qu’on pourrait peut-être encore trouver, des Allemand quivécurent à Litzmannstadt. Q, uels souvenirs ont-ils gue sordon Horwitz dont lombitiont que Goren paré dena de pas parle davantage des Tsiganes qui furent internés l'épidémie de typus [p 124] qui fut sans doute à l'oiine de leur assassinat à Kulmof On doute à lorigine de leur assassinat a Kulmhor. On peut signaler live (Micht Podeblebnik, Szymon Srebrnik) san live (Michal Podchlebnik, Szynon Srebrin) sont aussi des personnages du film Shoah de Claude Lanzmann. Beaucoup de personnes intéressées par un tel ouvrage ont sans doute vu lœuvre de Lanzmann de ces homm. ces hom Son sujet n'etait pas, en effet, de traiter de l'histoire de de mise à mort des Juifs nog quievoquentce centre de mise à mort des Juifs ne sont pas particulièrement approfondies. L'ouvrage néchappe pas à une sorte de loi a propos de lhistoire des centres de mise a mort dontìne

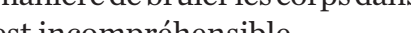

Il reste que le livre de Horwitz est exceptionnel. C'est certainement un des livres les plus forts qu'il soit donné de lire sur la Shoah, par labondance des ainsi que par la puissance de l'ém tonn'il lecteur insi que par la puissice de len Jean-François Forges

\section{LA CONCURRENCE \\ MEMORIELLE}

offrey Grandjean, Jérôme Jamin

Paris, Armand Colin, 2011, 256 Jérôme Jamin, rassemble des propositions de Jérôme Jamin, rassemble des propositions de
chercheurs qui travaillent sur la concurrence chercheurs qui travaillent sur la concurrence nant pour la cohésion sociale de nos sociétés.

Dans l'introduction, Geoffrey Grandjean pose les et de ses célébrations en affirmant qu'elle « entend d’abord préserver le sujet et le groupe [...], mais que la commémoration a perpétuité, loin de faire forcément advenir le bien, du a co du mal» [p. 6]. On sent dès lors toutel'importance associée à usage de cette notion. Lauteur installe le cadre dela rexion, focalisée sur la dynanique concurrenfois es questions d'effacement de comportant, à la foisles questions defacententect de conservation. Ces dernieres se nourrissent de la mémoire individuelle mais peuvent aussi être influencées par les priorités pour

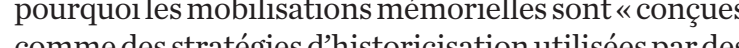
conteurs afin de produire du consensus ou dur acteurs afn de produire du consensus ou du désaccord pour la poursuite dobjectifs politiques » [p. 12]. une cost lorientation politique qui engendre, de fait, de des enir de certains faits historiques.

Dans cet ouvrage, plusieurs thèmes liés à la mémoire sont abordés : la Shoah, le processus de production de lois mémorielles, les multiples «émetteur d'histoire » [p. 17], la transmission intergénérationelle ou encore le rôle de l'école.

Dans la première partie intitulée «État de la question », Régine Robin s'est intéressée à la France et à son impossibilité dassumer son passé. En effet, elle la force du pays, «glisse vers la stigmatisatio, qui fait la force du pays, «glisse vers la stigmatisation des comnunautés » [p. 24]. Lauteure souligne que la mémoire nationale n'est finalement que la fragmentation de la méristion se résume en une « intégration par l'amour d'une nce plurielle» [p. 37].

Quantà Philippe Raxhon, il s'est penché sur la mise enperspective historiographique des lois mémorielles. Lauteur opere unedistinctionentrel'stoine connais-

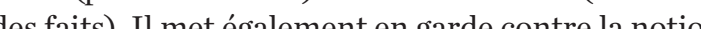
de mémo. de mémoire quipour lui est «une reconstructiond une

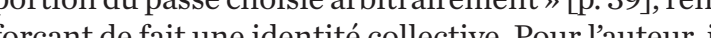
forçant de fait une identín collective. Pour lauteur, (lacomón définit de façon tout à fait cla. Il proporent

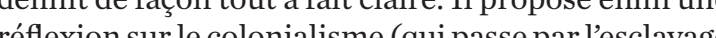
en l' puistimmigration) ensoulgn (qu passe par lesclave perisheris Sophis

Sophie Enst travaille sur les enseignement colle com Notre de la mémore et des détoun de la mémoire et des détouninents pervers des lauteure ilestnécs a lautenre, ilest assez rićne et puissantepour porter l'curage espoir dans lapro [p. 73] afin qu'il y ait réflexion sur les con sutes p. 73] afin qu'il y ait une reflexion sur les conduites dernier point que, de nos jours, il est difficile d'e derier point que, de nos jours, il est difficile d'éduquer et daccompanner la formation morale et civique de la jeunesse.

Dansla deuxième partie, «Études de cas», Geoffrey Grandjean présente son étude portant sur la diversit des memoires et la concurrence dans les discours liés à l'evocation de génocides chez les adolescents belges. L'auteur détaille le processus de son analyse en expliquant la mise en place des differents focus groups, qui sont des groupes de discussions explorant un ensemble spécifique de questions, mais également le rios propośs, questions posées en directet sceñnarios proposés, questions poseees en direct et enfin un de son étude: la première cst cue lesjeunes dorigine

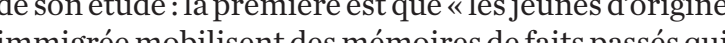
immigrée mobilisent des mémoires de faits passés qui leur sont proches à des fins revendicatives" [p. 112], et la secóde est relative au traitement différent de ces aits genocidaires entre les médias be pays d’origine des jeunes sondés.
Luis Bouza Garcia s'est focalisé sur les registres de mobilisation mémorielle dans l'espace public eurol'auteur, les références un tabou dans l'Union européenne puisqu'il existe des cotion

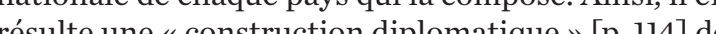
l'Union

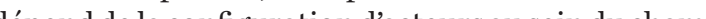
pelitique européen politique européen. Lauteur explique d'une part, que instrume de lhistoire à des fins de legitimation est un

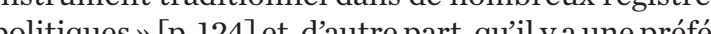
pelitich pour les logiques consensulles de l'oub

Gielia Fabling

Giulia Fabbiano quant à elle, s'est intéressée aux t'écriture de cette hèrtage et enprunts. En effet, lecriture de cette guerre "ńa pas su ou náa pas pu trouver une expression apaifre et dépassionnee $"[\mathrm{p} .131]$, ce qui a engendré une re dence len

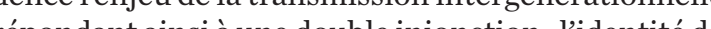
ivinte

Enf Śblie de songroupe de referen.

Enfin, Sébastien Boussois s'est penché sur les nouveaux historiens de lhistoire disraèl qui sont auss

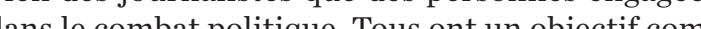
dans le combat politique. Tous ont un objectif commun, celui de mettre ajour « les preuves et l'origine d de l'exode des Palestiniens, dont certaines thè à so demisede

Dans la dernière partie, «Études connes.

Dans la dernière partie, «Études connexes », Joë Kotekévoque «le génocide paradigmatique»[p.167] de la Shoah. Il le définit comme incomparable, singulier, d'un précédentet commele refus du droit à lexistence dun groupe humain. Il en conclut alors à la fin de son explication quil sagit dun «crime purement idéologique et totalement gratuit pour ne répondre à aucune ouique guerrierc, tributive, utilitaire Serge Breduta » [p. 184].

Ses faux souvenirs via une analyse cognitive et notamment par le temoignage oculaire. Il explique les procédures pour chologie couxitive, dont les trois conditions majeures chologie cognitive, dont les trois conditions majeures nement, l'acceptation de la plausibilité de l'événement 
et une erreur d'attribution de la source d'une représentation» [p. 193].

Dans la conclusion, Jérôme Jamin souligne que la concurrence mémorielle expose la dimension imaginaire des sociétés et quel'histoire est présentée comm réation sociale et comme mobilisation à des fins politique, ethnique, lucrative et identitaire. Il met également en exergue les deux enjeux majeurs à ce couple histoire-mémoire D'un côté, «l'État doit assume le caractère construit de l'histoire de toutes société humaines tout en légitimant sa propre subjectivité. De l'autre, « les médias n'obéissent à aucune règle e matière de gestion du flux d'images quotidien, mai (tructurent l'ogenda des conflits» [p. 202]

Cet ouvrage met ainsi en évidence le fait que la concurrence mémorielle ne doit pas être considéré comme négative, mais comme la gestion du poids ménoriel decertans passés. Cestpourquoíl apparait bescó de

Anthony Michel

\section{LAFFAIRE KASZTNER. LE JUIF QUI NÉGOCIA AVEC EICHMANN}

adislaus Löb

Waterloo, André Versailles, 2013, 302 p.

'action de Rezsö Kasztner, Juif hongrois, commence lorsque Joël Brand est envoyé par les nazi en avion à Istanbul pour concrétiser la proposicontre 1000000 Juifs hongrois, soit un camion militaire pour 100 Juifs.

Les Anglo-américains, qui ne veulent même pas Les Anglo-americains, qui ne veulent même pas Eichmann de suspendre les déportations, empêchent Brand de retourner en Hongrie. Kasztner, quant à lui, devient l'interlocuteur de Eichmann pour essayer de sauver les Juifs hongrois de la déportation et de la mort, connaissant précisément leur destin.
Finalement, Kasztner arrive à organiser un train vers la Suisse avec 1684 personnes, parmi lesquelle vers la Suisse avec 1684 personnes, parmi lesquelle prose probable que Kaszter soit parvenu à sauver dautres Celive, relat nentes recte aucune preuve.

Ce live, relatant les tractations de Kasztner avec les nazis qui sapparentaient plutôt à des parties de poker ment Kasztner pr. Posait $100 \$$ par tête, les naz personnes, Kasztner proposait 100 \$ par tête, les nazis locaux en voulaient 2 000. Finalé. $\$$, ce qui fut paye.

(loctations : lorsqu'il lui appart que léchange « camions contre Juifs »était un echec, il proposa un échange contre des matières prenié Lés var les Anglo-américains.

Les verbatin des carnets de Kasztner décrivent sesirente trévèlent le caractère ambivalent dece personnage ainsi que létendue de son pouvoir. Il nétait pas, en effet, comme ill'a déclaré lo

Le rôle du « Judenrat « que les Allemands inpo-

Le rôle du « Judent saient auxcon usu 1684 equivoque, mais la constitution de la liste de 1684 personnes montre combis choisir qui sauver

L'affaire Kasztner. Le juif qui négocia avec Eichmann décrit aussi les massacres et noyades massifs de Juifs auxquels se sont livées les "Croix fêchées » qui avaient pris le pouvoir après la destitution d'Horthy par les Allemands (qui leur reprochaient uniquement de laisser les cadaves sur place, et les corps des noyé

Les héritiers politiques des "Croix fléchées 》 constituent de nos jours la $3^{\mathrm{e}}$ force politique hongroise tiobbik a obtenu $17 \%$ des voix lors des dernières élections. Les "Croix fléchées " avaient réalisé le même $3 \%$ de moins que le parti socialiste.

Henri Goldberg

\section{LA VOIE DES IMAGES. QUATRE HISTOIRES DE TOURNAGE AU PRINTEMPS-ÉTÉ 1944}

Sylvie Lindeperg
Lagrasse, Verdier, coll. « Histoire », 2013, 282 p.

Q

faire des images filmées du passé, depuis lus d'un siècle que le cinéma existe? Plu roches de nous, que faire de ces images tournees il y a plus d'un demi-siècle, images de la guer et des génocides? Comment les cinéastes ou auteurs d'aujourd'hui? Comm inserent-ils dans leurs œures d'aujourd hui ? Comment les spectateurs et téléspectateurs les reçoivent-ils? Sagit-ilde combler la distance

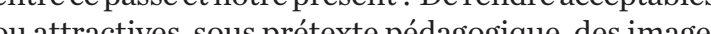

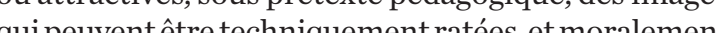
quipeuvent êtretechniquenentrates, et moralement afreuses? Et de façon plus générale: ce passe à la for lointain et proche, que peut-on en faire, qua-t-on ,y a-til pas une hiérarchie átablir? Cest as une questions que Sylvie Lind

Cest a ces questions que Sylvie Lindeperg se propose de réporions del'année 1944 to delannée 1944:toun gestans le nuis du Vercors, derezin

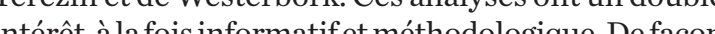

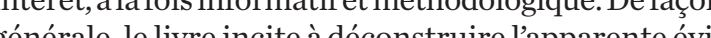
gênérale, lelivie

Exemplaire à ce propos, la mise au point sur la célèbre photographie du Vel' d'Hiv, montrant des femmes assises ou couchées sur la piste du stade, soudes Juifs en juillet 1942 Or elle représente en fait des cos Juirs en collaborateurs internés au mene endroit entre le Sout en 2 septembre 1944, comme la démontré rerge Klarsfeld. Hn'existe donc aucune image historique de la rafle du Vel' diviv. Le film La Rafle (2010) Cuichets du Loure (1974) ou. Monsieur Klein (1976). Guichets du Louvre (1974) ou Monsieur Klein (1976). Cest surtout sinscrire dans une logique " empaLa Rafle vide l'histoire de sa subtan à juste titre de sofintelligibité pour nen conserver queléchet émotionnelle l'édification morale, la privatisation des luttes collectives et la compassion pour les victimes. Et de rappeler que le «devoir de mémoire» «procur $[e]$ bienfaisant confort moral, clos sur lui-même, privé introspection sur le présent, dépouillé de sa responsabilite à l'egard du futur. » Le Vel' d'Hiv fut encore Surlásés Surla Résistance, peu d hinages au départ, pour des de la Lintes de sécurité. En 1944, à lapproche destinés aux Alliés - puis des « clandestins ont lieu, destinés aux Alliès - puis des « images d'après coup »,

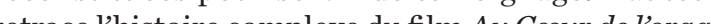
de plusieurs ros, sur les maquis du vercors, réerit à Comité de libération du cinéma français (CLCF), des TTP des gaullistes, et qui utilise des scènes tou, des Tre des gaulistes, et qui utilise des scènes tournées En revanche, le film La Libération de Paris, tourne

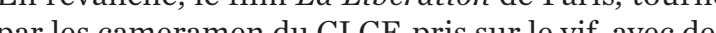
par les cáels mon due leur déroulement: mais la bande-son u qui reprodut ences

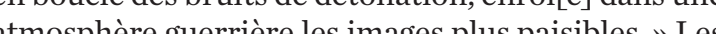
cosper tion de soldats allemands, des femmes tondues; tains de ces derniers plans, tournés par des am ; fure déc dés au montage, de même que les imars. des enipers qui tirent sur la foule rase qués a Dame. Ces ima tres revanche furent récuṕ, Dame. Ces images en revanche furent recuperees par brutalités de l'épuration, pour

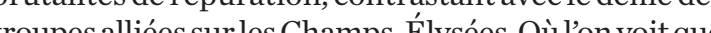
troupes alliées sur les Champs-Êlysées. Oủ on voit que image, qưon peutcroirenaivenent «objective», peut dictor des massages politiques précis et parfois contra-

Par la suite, les producteurs utilisent des plans empruntessàdesfictions d'apres-guerre (UnAmi viendra (pour La bataille durail) ou à des scènes reconstituees (pourle Vercors, Au Coeur de lorage).Ainsil 'exécution tée » par la scène équivalente du film La Bataille du rail (1945). ils l'auraient fait différemment : a car l'archive filmée s lauraient fait différemment: «car larchive filmée est aussi une archive de manières de filmer. "

De cette «nanière de filmer », les deux toumages, celui de Terezin et celui de Westerbork, offrent deux extières écalement suggestifs, mais sans doute pas 
taires. Le film de propagande sur Theresienstadt fut tourné par une équipe d'internés juifs, sur l'initiative

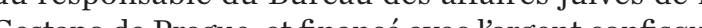
Gestapo de aux Juifs tcheques. Il sagissait de presenter Terezin comme une villegiature paradisiaque et de dissimule Croix Croix-Rouge,le Vatican, les pays neutres. Aucune copie conplente arch . Jude (1940), torné dans cest Thverse de Der Ewige

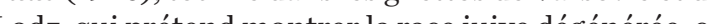
Lodz, qui pretend montrer la race juive dégénérée, ce qui est avant tout le resultat des persécutions nazies. Auchor tiques, sportís et travailleurs. Peu exploite, il est surLinderis méco con Lindeperg ezardent le masque de la propagande.

Eneffet, al interins internés tentaient de saboter limage programmée par les hazis. des dessinateurs, qui produisent pour la propagande des chromos idyli dues du canp, font aussi des eures clandestines quila dénoncent, et que souvent ils paieront de leur vie. Le film ne pouvait évidemment a ari qui sont saisies par le film: hymne national allemand récrit en mineur, hymne national tchèque dissimulé ansle contrepoint, plusieurs is os du Kol Nidréqu signifieraient: «que ces images ne soient pas regardeees comérité

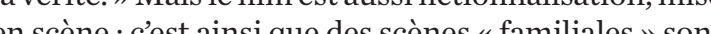
en scène : c'est ainsi que des scènes «familiales » son nautés juives de Berlin et d'Amsterdam, déportées nrès, après, etcechoix, «négigence, inconséquenceoulapsus,

En fait Theresienstadt reconduit deux tr. En fait, Theresienstadt reconduit deux traits majeurs de la machine genocidaire nazle :la conjonccion entre la mise à mort et sa dissimulation, entre crime et son écente cont inscite au cœur du tourage, la volonté de contraindre les Juifs à participer au d’ordonn dexterninatén séaliser eux-mêmes le filn dordonner aux de propagande.

sur Westerbork, tourné entre mars et mai 1944, et consistant en 95 minutes de rushes muets, obéit à un autre projet : en 1944, la majorité des Juifs hollandais ayant été déportée, le commandant du camp, Gemmeker, veut transformer le camp en Arbeitslager, ce qui lui éviterait d’être envoyé sur le front de l'Est. Du coup, le film, reprenant les codes du «film d'entreprise», insiste sur le travail des internés dans les ateliers, consacre par exemple quinze minutes sur la chaîne de recyclage des moteurs d'avion. Les divertissements ne sont pas oubliés : comme à Terezin, les internés présentent des spectacles de cabaret, mais qui ont souvent lieu le soir même des départs de convois, comme le montre entre autres le témoignage d'Etty Hillesum. Et ce sont les séquences des convoi qui seront projetées au procès de Gemmeker, comme pièces à charge.

Ces séquences frappent aujourd'hui par leur déroulement paisible : ceux qui montent dans ces wagons, savent-ils où ils vont ? Ou sont-ils rassurés par la présence de l'opérateur? Resnais visionne ces rushes en 1955 : il prend conscience de leur importance, sans savoir encore qu'il sagit des seuls plans connus montrant le derpart d's convoi vers l'st depuis lEurope foulard foulard blanc, dans lentrebâllement du wagon, devenue une icône du judéocide. En 1994, au terme d'une enquête, elle est juve, liviée : elle était sinti et non pas juive, « lemblème d'un génocide sous-exposé dan

Impossible dans l'espace d'un compte rendu d'entrer dans les précisions des details techniques, qu pourraient paraittre fastidieux, mais se revèelent passionnants par tout ce quilis font émerger - du geste jeurd'hui. Commel'écrit Jean-Luis Coctateur daujourd hui. Conmelecrit Jean-Louis Comolli dans son dialogue aveckauteur, «ilneviendrait pas al esprit d'un été employé dans la guerre bes camérarmement qui éte enoto lenoir et blanc,labsence de son, voila descirconstances historiques qui nous en disent autant sur l'epoque et la guerre que les détails des armes ou des uniformes. » Coloriser ces archives audiovisuelles, au prétexte que le spectateur naime pas le noir et blanc, les sonorise quandello Cestes que le cinéma est hors histoire.

Certes, il dest pas pous de de faire abstraction de

Le spectateur d'aujourd'hui ne peut faire autrement que de peupler ce hors champ de cadavres, non promis dans

le champ. Mais il est non moins indispensable de garde présent à l'esprit notre distance temporelle, pour ne pas céder à ce que l'auteur appelle l'esthétique du trop-plei et de l'hypervisibilité ; chevauchement et hybridation des ấges et des régimes du visible, [...] pulvérisation des durées et nivellement des temporalités.

À cette condition, l'image peut nous parler, et l'auteur rappelle le conseil de Schopenhauer à un étudiant teur rappelle leconseilde Schopenhauer àun étudiant en à pii cor'il âi,ces livines

Anne Roche

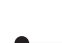

A DERNIÈRE CATASTROPHE.

L'HISTOIRE, LE PRÉSENT,

\section{LE CONTEMPORAIN}

Henry Rouss

Paris, Gallimard, coll. « Essais », 2013, 352 p.

$\mathrm{N}$

au Caire en 1954, Henry Rousso appartient e génération d'historiens de «l'après », une génération pour laquelle la « dernière catastrophe », au moment où il fait ses premiers pas en tant que chercheur, est la seconde Guerre mondiale, Un Che porteront dailleurs ses premiers travaux [Un Cha Paris, Ramsay, 1980], avant qu'il ne s'impose dans panorama universitaire français et etranger, avec ses ouvrages sur le régime de Vichy, comme lune des coures inconton n'hésitant pas à lever des tabounce contenporane, nîssitant pas à lever des tabous, explorant sans relâche les aspects les plus obscurs d'u "passé quine passe pas» [Le Syndrome de Vichy 1944 198..., Paris, Seuil, 1987, La Collaboration. Les Noms, Vivre sous l'Ocux, Paris, MA, 1987, Les Annéesnoires. Vivresous LOcupation, Pars, Gallinard, coll. «Découvertes », 1992; Vichy, unpassé qui ne passe pas, avec Eric Cona, Pans, Fayar, 1994.

Lepoint de départ de son dernier ouvrage, au début étiez pas ! », relate un épisode qui pourrait s'apparenançois Bédarida, alo , âgé d'une soixantaine d'années et ayan d'un différend cériode de loccupation, qui, à l'occasio ur le cim dich du lempés

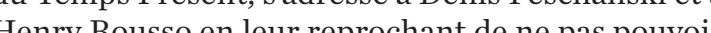
Henry Rousso en leur reprochant de ne pas pouvoi lité, le nens de l'anecdote va bien au-dela des rétites ivalités versitaire ils'y exprime au fond un probe raphique qu sera au cour d'une profonde réfexion graphique qui sera au cœur d une profonde réflexion menée par historien dans son ouvrage, la question de à contempor les présupposés de l'histoire dite a ducher arésent l'oust-ce donc que l'histoire contemps présent ». Qu uest-ce donc que l'histoire contemporaine, immediate ou du temps present, appellation concurrentes - quoique pas exactement synonyme pour désigner une partie de a « discipline historique [p. 13]? qulle est la question, da marge au centre p. 13] Telle est la question à laquelle louvrage de Henry Rousso s'eforce de répondre en 266 pages d'une projet relevait d'une sorte de " manifeste ofe so projet relevait d'une sorte de "manifeste offensif [p. 23], illa la denent dépassépour se transformer en tes

L'ouvrage se structure en quatre grands chapitres qui abordent la question sous des angles différents. Le premier d'entre eux recherche «la contemporaneite dans le passén, c'est-à-dire la manière dont les historiens du passé ont pu penser - ou pas - le present ou depuis l'Antiquité, le statut du contempoin les ouv lAntiquité, le statut du contemporain dans Prost, cité pritos. est une vieille histoire ! L autreme dut que present pour con pour la contemporaneité n'est pas l'apanage de notre la Révolution françise pour que con d'histore la Révolution française pour que le concept d'histoire XIXe siècle une rupture nette entre possé to prés Au moment même où la notion de contemporanéite u moment même où la notion de contemporanéte sériocine dans lunivers mental du XIX siecle, cette période principe nouveau du regard distartique, en vert du principe nouveau du regard d'stant qui invalide le 
même de l'histoire comme discipline autonome rend du coup suspecte une histoire contemporaine qu jusque-là, faisait partie intrinsèque du regard et de

pratique des historiens » [p. 65].

Au chapitre deux de louvrage, l'auteur met en avant le changement de sens qui, affectant la notion de contemporaneite a partir de la Premiere Guerre Lévéne bou vions a Léveénenent, dans sa violence et sa soudaineté, cree au cœur mène de la défagration, puis dans son après-coup, lé sentinent d'une nouvelle rupture dans différence avec la ucue $₫$ [p. 87-88]. Mais la grande différence avec la " catastrophe davant », c'est que, un futur tourné vers le progrès, la Grande Guer cetteidée d'une raison est réféfi : engé il met son pu re

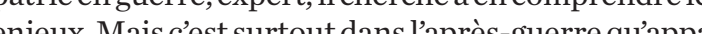
jeux. Maiscest surtout dans lapres guere quappapaissé, denour passé, mar ctc. qui configurentle socle d' émoration púm (1920-1930) Ar laurice Hal-

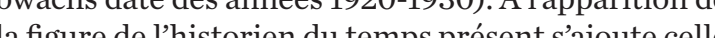
a figure de lhistorien du temps présent s ajoute celle taire pour dire l'histoire: Leur compagnomplémentaire pour dire lhistoire. «Leur compagnonnage, leu du XXe siècle un élément cont constel l'éc tout au lon tore tra toire tragque du temps present " [p. 99]. Ir en ira de prene de la Seconde Guerre mondiale, mais le choc

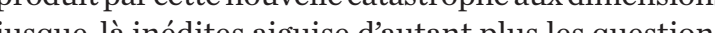
jusque-lá inédites aiguise dautant plus les questions soulevées par la Première. L'apparition d'une judiciarisution internationaux (Nuremberget Tolyo) ins tribua [p. 127] avecle droitet la justice, qui pet contictuels p.127] avecle droit et lajustice, qui pèseront lourd su l'histoire de cette présent. Enhere Rousso montre que Césynchronisé qui génère des «efe ts rề à un temps désynchronise qui génère des « effets retard »: ce passé traumatique va occuper à la fin du XXe siècl d’avoir en 1945. Les représentants de la génération d'après héritent d'un traumatismetransenetration tenu d'une catastrophe qu'ils n'ont pácue

Le tro eatastrophe qu ilsn ont pas vécue» [p. 143].

Le troisiène chapitre est consacre au phenomèn l'institutionnalisation progressive de la contempora- néité. Il s'agit bien là d'un véritable tournant, voire d'un «retournement» [p. 145] puisque, occupant une place mineure jusque dans les années 1970-1980, le temps présent va s'imposer jusqua a devenir, de nos jours, le "régime d'historicité dominant », et ce, non seulement au sein de la discipline historique, mais aussi, ins largenent dans le channp social et culturel où les images des catastrophes qui se sont succédé depuis la trande G'un idtité collective qu « force strucpériodes pasées, ou plus autant que n'ont plus les périodes passées, ou plus autant. Le chapitre caractérise ce bouleversement qui s'impose de manière plus ou moins tardive dans les pays considerés pa Rousso, par exemple pour lAllemagne, qui fait figure de pionniòre avec la création en 1945 de linstitut fü Zeitgeschichte, pour le developpement de l'histoire du national-socialis l' Notons que se produit en parallèle lémergence d'une forte demande sociale, qui se traduira par une présence indéniable de l'histoire contemporaine dans les médias de masse. En France, il faudra cependant attendre les années 1970-1980 pour que les réticences épistémologiques finissent par tomber.En effet, Implantation du paradigme de la longue duree, en particulier autour de Fernand Braudel, ouvre certes sur un nouveau rapport entre passé et présent, mais fait en mene temps obstacle au développement autonome delhistoire contemporaine qu'il retarde par

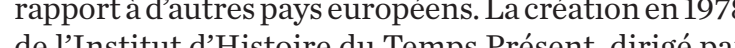
de linstitut d Histoire du Temps Présent, dirige pa François Bédarida puis par Henry Rousso lui-même au tournant du millenaire, peut être considérée d'une certaine manière comme le couronnement de ce processus d'institutionnalisation de la contemporanéité Méné si, lanalyse fort bien lauteur, sa création obéi au départ a un besoin d'abord diffus. Ce n'est que dans le sillage de cette création que les enjeux propres à la contemporaneite en histoire vont surgir et conduire fuussi la pace de l'hsist non seulement l'histoire, mais Ce place de lhistorien dans le monde.

Ce sont précisément ces enjeux que le quatrième et dernier chapitre, « Notre temps », va poser. Henry Rousso tente de cerner les contours de ce succès de lhistoire contemporaine, dont les multiples cause visuelle pogees tour a tour: explosion de loffre audio-

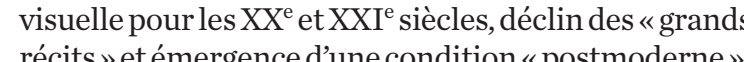
récits » et émergence d'une condition «postmoderne», «tournant langagier » conduisant à la remise en cause
des modèles dominants, fin du paradiome national avec l'ém don d'u , fin du paradigne national pour reprendre les analyses de François Hartog, l'avènement d'un régime d'historicité « présentiste » dan an contexte de crise de l'avenit. Les deniters souschapitres de louviage n'occultent pas les linites de C'est int constituéconme Cest ainsi que, dans la partie intitulée "Appellations plus ou mons controlés n, l'auteur met en évidence dans «Une définition par critères variables", il

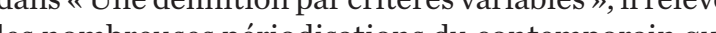
ont pu être proposées, allant de 1789 à 1989 (chain qu (1) Cor Silest Center).Silest peut-êtreprécisénentunélément qui

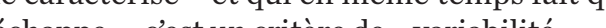
échappe-, cest un critè me uvariabilitè».

Aut total, Pouvrage de Henry Rousso présente moin une histoire du temps présent qu'il n'en met en évidém le de tout his

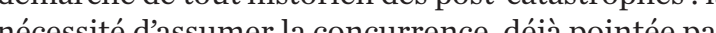
nécessité dassumer la concurrence, déjà pointee par Braudel, entre « deux nécessités épistémologiques contraires », celle de la « mise à distance » et celle

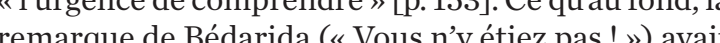
mis au jour chez l', historien un peu im ! [p.14] ju'teit Rousso historien un peu immature [p.14] quétait Roussoen nement confirmé consacrera un ouvrage plus de ving ans après. On pour d'autres formes, est présente dans tonné toriqu, ycompis des periods largentanter car elle n'est au fond qu'un avatar de l'effet de projection autobiographique propreà toute appréhension de Si l'e d'Henry Rousso in clut des rérences Si l'essai d'Henry Rousso inclut des référence bien sûr françaises, hais aussi allemandes ou anglofor quisestmón

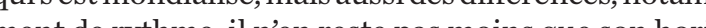
ment de réflexion il n'en reste pas moins que son horzon de admet fort bien. Il serait interessant de confronter es cours cours des deux siècles derniers, ce dont leurs historio-

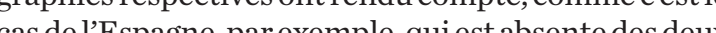
cos del Espagne, par exemple, qui est absente des deux cour

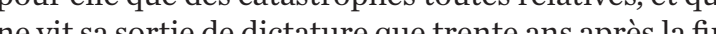
de la Secor le la même manière au cours du XXe l'avènement d'un régime d'historicité prés seccle, lavènement dun régime dhistoricité presentiste, férent, ainsi que ses enjeux spécifiques, déterminés pert, ainsi que ses enjeux speciliques, deternines Mais l'ouvrage d'Henry Rousso ne caurait qu'en nourri d'autres, qui drenry différes, qui prendrónt le relais pour comprendre les diférentes modalies de laven pour cont de lhistoire du 